\title{
Free Tissue Transfer
}

National Cancer Institute

\section{Source}

National Cancer Institute. Free Tissue Transfer. NCI Thesaurus. Code C148436.

The transplantation of autologous tissue that is completely detached from its original blood supply from one location to another using microvascular reconstruction techniques to reestablish circulation. 